\title{
Dynamic simulation of palletizing robots based on ADAMS $^{1}$ RUI Zhiyuan ${ }^{1 \mathrm{a}}$, ZHOU Baocheng ${ }^{2 \mathrm{~b}}$ LIU Jun ${ }^{3 \mathrm{c}}$ \\ (School of Mechanical and Electronic Engineering, Lanzhou University of Technology,Lanzhou,730050,China) \\ aexeter_lut@126.com, ${ }^{b}$ woxinyijiu.870116@163.com, Izhjliu@126.com
}

Key words: MOTOMAN-EPL300 palletizing robot;dynamic analysis;ADAMS

\begin{abstract}
This paper studies the special aluminum ingot palletizing robots for casting machines. It established the robot model with the three-dimension model-building software Pro / E and analyzed and simulated the dynamics of palletizing robots with the software ADAMS to achieve the dynamic characteristics curves of the robot joints speed, displacement, etc. The simulated results verify the accuracy of the dynamic mathematic model, providing theoretical basis for further research on dynamic characteristics of palletizing robots.
\end{abstract}

\section{Introduction}

In the last twenty years, robot technology has developed very quickly which is a comprehensive technology integrating mechanical technology, automatic control technology, computer technology, artificial intelligence, microelectronics, optical, communication technology, sensor technology, bionics science, etc. It represents the frontier of high-tech development. It is a popular direction of the current scientific and technological research. Palletizing technology is a new technology in the field of logistics automation technology. Palletizing means the adoption of the idea of integration unit to stack the materials according to certain models to realize logistic activities such as storage, handing, loading, unloading and transportation of the materials units ${ }^{[1]}$, In recent years, with the development of the national economy, expansion of production scale and improvement in the level of mechanical automation, stacking technology, especially palletizing robot technology, is more and more extensively applied and is leading the trend due to its advantages in the mechanical structure, scope of application, the device footprint, flexibility, cost and the maintenance. So far, palletizing robot is widely used in the pharmaceutical industry, petrochemical industry, food, household appliances as well as agriculture and many other areas. Therefore, it is meaningful to do research on palletizing robots' movement characteristics and their execution accuracy and stability ${ }^{[2]}$. The structure of the MOTOMAN-EPL300 palletizing robot concerned in this paper is shown in Fig.1 below:

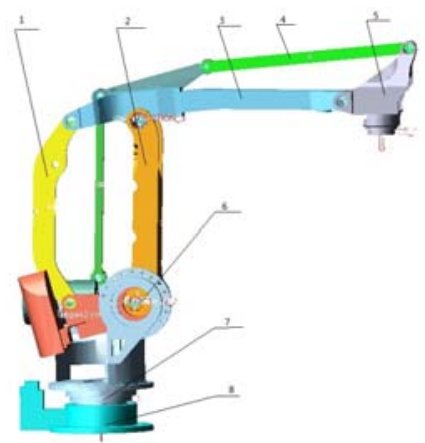

Fig.1
1. Connecting rod 12. arm
3. Forearm
4.connecting rod 2
5. Wrist
6.motor
7.Waist turntable
8.foundation 


\section{Main technical parameters of MOTOMAN-EPL300 palletizing robot are researched in this subject}

The palletizing robot is designed by our Research Group, it is reference MOTOMAN-EPL300 vertical multi-jointed robot, produced by Shougang Motoman Robot Co., Ltd. The robot is designed with advantages of high transmission accuracy, compact structure, large workspace, good adaptability, can be widely used in palletizing, handling, arc welding, spot welding, and other field. This robot body structure mainly consists of the waist, arm, forearm and wrist and end actuators five parts. It has four rotating joint, four degrees of freedom.

According to the job requirements of the palletizing robot for aluminum casting production line: The speed of palletizing 9.6/cycles, palletizing weight is $300 \mathrm{~kg}$, vertical distance $2500 \mathrm{~mm}$, level distance2300, the distance between end actuator and aluminum layer is $1000 \mathrm{~mm}$, when machine in the initial position. One work cycle of palletizing consists of: Aluminum Grab, aluminum rotation, aluminum stacking and back to initial position four parts.

(1) Aluminum Grab: First, Grasp from the initial position to the aluminum layer needs 1.6s. Then grab aluminum needs $1.6 \mathrm{~s}$, then back to the initial position need 1.6s.

(2) Aluminum rotation: the aluminum ingots rotate $90^{\circ}$ in $1.6 \mathrm{~s}$ around the axis of waist, meanwhile grab aluminum ingots to stack position.

(3) Aluminum Stacking: The end actuator move down $1000 \mathrm{~mm}$ in $1.6 \mathrm{~s}$, reach aluminum stacking position, and then aluminum is placed in order within 1.6s.

(4) Return to the initial position: end actuators up $1000 \mathrm{~mm}$, and then rotate $90^{\circ}$ around the axis of the waist to the initial position.

In this paper, according to the robot structure, use Pro/E software established three-dimension entity model of the MOTOMAN-EPL300 robot, and its overall structure as showed in fig.1, the main structural parameters shown in figure 2 and 3, the main technical parameters of the robot as showed in table 1

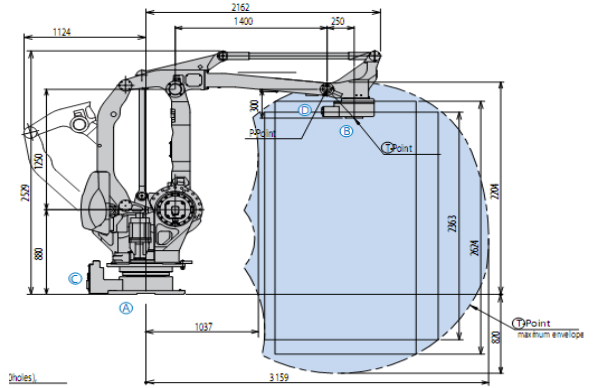

Fig. 2



Fig. 3

Table 1 MOTOMAN-EPL300 palletizing robot main technical parameters

\begin{tabular}{|c|c|c|}
\hline \multicolumn{2}{|c|}{ type } & MOTOMAN-EPL300 \\
\hline \multicolumn{2}{|c|}{ Structure } & vertical multi-jointed \\
\hline $\begin{array}{c}\text { Ciggest } \\
\text { action } \\
\text { range }\end{array}$ & $\begin{array}{c}\text { S-Axis(rotation/lateral } \\
\text { swing) }\end{array}$ & 4 \\
\cline { 2 - 3 } & $\begin{array}{c}\text { L-Axis(lower arm } \\
\text { tilting) }\end{array}$ & $+180^{\circ}$ \\
\cline { 2 - 3 } & $\begin{array}{c}\text { U-Axis(upper arm } \\
\text { tilting) }\end{array}$ & $+90^{\circ} /-45^{\circ}$ \\
\cline { 2 - 3 } & B-Axis & ------ \\
\cline { 2 - 3 } & T-Axis(circumgyration) & \\
\hline maximum & S-Axis & $\pm 180^{\circ} /-120^{\circ}$ \\
\cline { 2 - 3 } speed & L-Axis & $90^{\circ} / \mathrm{s}$ \\
\cline { 2 - 3 } & U-Axis & $105^{\circ} / \mathrm{s}$ \\
\cline { 2 - 3 } & B-Axis & ------ \\
\hline
\end{tabular}




\begin{tabular}{|c|c|}
\hline T-Axis & $195^{\circ} / \mathrm{s}$ \\
\hline Robot weight & 1,820 kg(4,013.1lbs.) \\
\hline vertical distance & 3,024 mm(119.1") \\
\hline level distance & 3,159 mm(124.4") \\
\hline Braking mode & All axis \\
\hline $\begin{array}{l}\text { Allowance of the moment of } \\
\text { inertia(GD2/4) }\end{array}$ & $80 \mathrm{~kg} \bullet \mathrm{m} 2$ \\
\hline
\end{tabular}

\section{Palliating robot simulation and motion analysis based on ADAMS}

The goal of palliating robot simulation and motion analysis based on ADAMS. In order to analyze the robot displacement, velocity, acceleration, force and moment relationship ${ }^{[3]}$. And take advantage of this through the various executive joint rotation, to better control the robot and wrist pose. Using ADAMS analysis robot kinematics simulation and analysis, one of the biggest characteristics is intuitive; it is convenient for controlling the movement interference ${ }^{[4]}$. The questions are simulated and analyzed as following: in motion process observation whether movement is feasible, whether interference; observe whether manipulator arrived to a designated position for grab aluminums ingot when sports end. Because ADAMS software entity for three-dimension modeling functions is relatively weak, sousing PRO/E software established the pallet robot entity model. Robots entity Model import in ADAMS through the interface software Mechanical/Pro for Pro / E and ADAMS. Realized the import, Then parameter setting to model, drive loading and the joint rotation control constraint, the simulation model as showed in fig. 2:

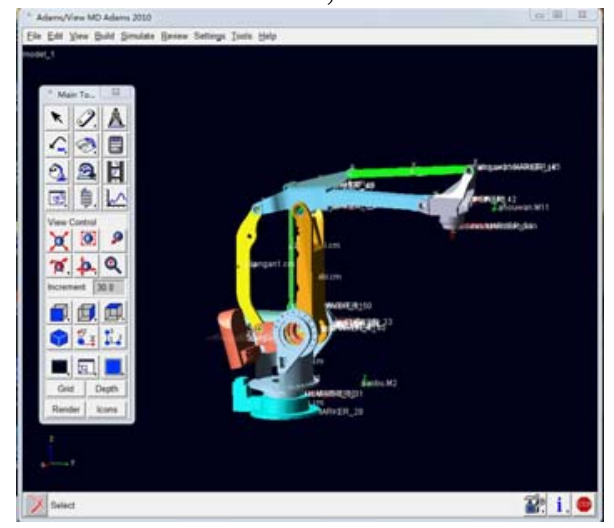

Fig. 2

The end point (moduandian) on the robot end effecter as measuring object, obtained the robot end effecter displacement curve, the speed curve, acceleration curve in X, Y, Z direction relative to base coordinate. Displacement curve as showed in fig. 3 ,The speed curve as showed in fig.4, Acceleration curve as showed in fig.5

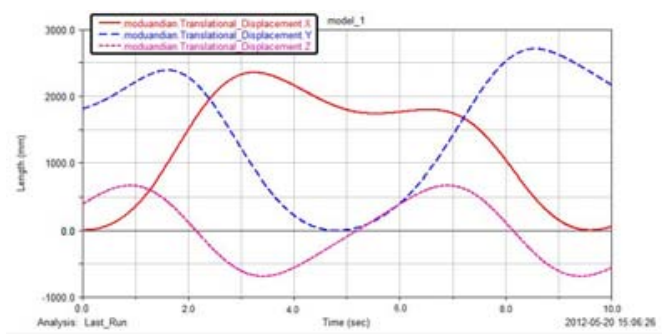

Fig.3

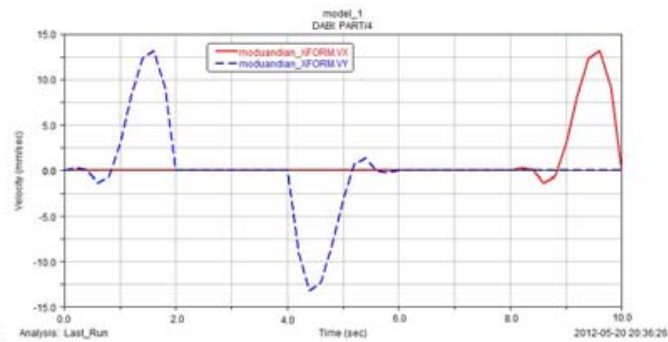

Fig. 4 


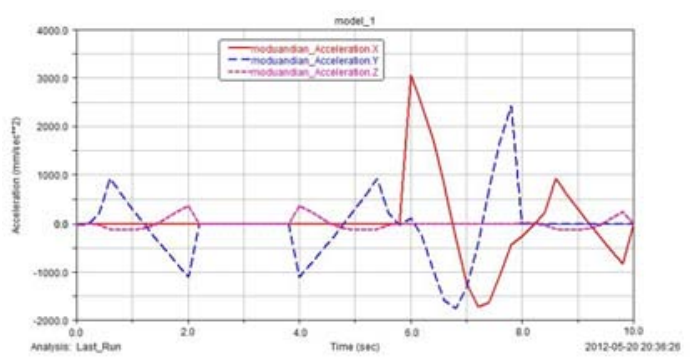

Fig. 5

\section{Conclusion}

As showed in fig. 3and5, the solid line is the displacement curve of the end point in the $\mathrm{X}$ direction, The point line is the displacement curve of the end point in the Y direction, dotted line is the displacement curve of the end point in $\mathrm{Z}$ direction. According to the "Plot Tracking" of Postprocessor (post-processing module) can measure displacement value accurately in the $\mathrm{X}, \mathrm{Y}, \mathrm{Z}$ directions :The maximum displacement in $\mathrm{X}$ and $\mathrm{Y}$ direction is $2995 \mathrm{~mm}$, the maximum displacement in $\mathrm{Z}$ direction is $2600 \mathrm{~mm}$, proved that the robot can meet the requirements of the work scope. The results of simulation analysis: observed the simulation of movement process , the whole process of grasping movement is feasible, movement interference did not happen; From the simulation figure can see, manipulator can satisfy grab task; The curve of hand origin coordinates and aluminums ingot distance changes with time display manipulator wrist can finally arrive at the target location. So, we can basically draw a conclusion that: each joint manipulator layout design is reasonable, manipulator kinematic algorithm is correct. The kinematics calculation module is applied to the control system of an experimental prototype, through the experiment, the robot can accurately catch the target, further proves that the algorithm is correct.

\section{References}

[1] ZengKongGeng, WangHongQing, DingYuanyan. High speed robot handling stacking system structure and technical [J].Robot technology and applications, 2001 (4)

[2] CAI zixing. Robotics [M]. Beijing: tsinghua university press, 2000

[3] YangRuQing, GaoJianGuo, HuHongGuo. High speed pallet key technology research [J]. High technology communication, 2004, 14 (1)

[4] ChenWenHua, Heqingchuan, ZhangDanwen. ADAMS2007 mechanism design and analysis example [M]. Beijing; Mechanical Industry Press, 2009.

[5] ChenXueSheng, Chenzaili, KongMinXiu, XieTao . parallel robot kinematics accurate solution based on neural network 6 - SPS [J]. Journal of Harbin industrial university,2002 (1).

[6] GuoWeiDong. Virtual prototype technology and ADAMS application example tutorial [M]. Beijing; The Beijing university of aeronautics \&astronautics press, 2008.

[7] GuoHongGong, HeJiLin etc. Industrial robot technology [M]. Xian; Xian university of electronic science and technology press, 2006. 